\title{
Effect of environmental atmosphere and stress-loading storage on the intrinsic stress and its distribution in polycarbonate screw cap
}

\author{
Qiang Yang ${ }^{a}$, Jun Liu ${ }^{b}$, Mingzhen $\mathrm{Li}^{\mathrm{c}}$, Guangping Tang ${ }^{\mathrm{d}}$ \\ Institute of Machinery Manufacturing Technology, China Academy of Engineering Physics, \\ P.O.Box 919-624, Mianyang, 621900, P.R.China \\ ayangqiang817@hotmail.com, ’dfh0070@163.com, chmhy1972@163.com, 'Banquer@163.com
}

Keywords: Polycarbonate, Screw cap, Environmental atmosphere storage, Stress loading storage, Intrinsic stress

Abstract: The polycarbonate screw cap part had cracking problem during storage, which mechanism needed to be studied. The effect of environmental atmosphere and stress-loading storage on the intrinsic stress and its distribution in polycarbonate screw cap part was investigated in the paper. The analytical results indicated that the intrinsic stress in polycarbonate screw cap had a tendency to decrease slightly or to keep unchanged during room temperature-low humidity $(40 \% \mathrm{RH})$ storage and room temperature-middle humidity (55-65\% RH) storage for 315 days, while had a tendency to decrease obviously during 315 days of high temperature-high humidity $\left(47^{\circ} \mathrm{C}+90 \% \mathrm{RH}\right)$ storage. After 300 days of stress-loading storage, the intrinsic stress in polycarbonate screw cap part also decreased obviously, and it might belong to stress relaxation. The distribution of intrinsic stress in polycarbonate screw cap part basically kept unchanged during environmental atmosphere storage and stress-loading storage.

\section{Introduction}

Polycarbonate (PC) screw cap part was an important part in certain product. When it experienced long-term storage, because of intrinsic stress concentration and other causes, a few cracks were found nearby a small screwed hole on the polycarbonate screw cap part, which seriously affected its usage reliability. Therefore it's needed to study the storage cracking causes and mechanism for polycarbonate screw cap, and to know how these intrinsic stresses change during storage. Environmental atmosphere and stress loading may lead to change of the intrinsic stress in the polycarbonate screw cap part. Therefore, we investigated the effect of environmental atmosphere and stress loading storage on intrinsic stress and its distribution in the polycarbonate screw cap in the paper.

Stress cracking (SC) and environmental stress cracking (ESC) are two main mechanisms for cracked polycarbonate parts [1-8]. Stress concentration can cause polycarbonate parts to crack directly, which is mainly related to properties of materials. And environmental stress cracking is caused by small stress while contacting chemicals or organic solvents (including gas), which mainly exist in usage environment. Both mechanisms have important effect on material's usage. Stress in different transparency materials can be determined by photo-elastic method [9-14].

In the paper, a batch of PC screw cap assembly parts were tested after stored in different environmental atmospheres, including room temperature and low humidity, room temperature and middle humidity, high temperature and high humidity atmosphere. Another batch of screw cap assembly parts were tested after stored in stress-loading state. The intrinsic stress and its 
distribution of each part were measured by photo-elastic method after certain period of storage. And the related changing trend and rules of the intrinsic stress and its distribution were analyzed and probed.

\section{Experimental}

\section{Environmental atmosphere storage experiment}

15 screw cap assembly parts were stored in environmental atmosphere experiment. At first the screw cap assembly part was assembled as initial state. Then a small pre-stress was applied on the screw cap part by rotating a screw tightly for $90^{\circ}$ from initial position, and then the intrinsic stress in the PC screw cap at the initial state were measured. The 15 screw cap assembly parts were kept at this state for storage. The intrinsic stress and its distribution in each screw cap assembly part were measured after certain period of storage.

5 screw cap assembly parts were stored in room temperature and low humidity (40\%RH) atmosphere, which was an environment inside a dehumidify cabinet (A20-290, Dr. Storage Co.). 5 screw cap assembly parts were stored in room temperature and middle humidity (55\%RH-65\%RH) atmosphere, which was a normal laboratory environment with dehumidifier running constantly. And 5 screw cap assembly parts were stored in high temperature $\left(47^{\circ} \mathrm{C}\right)$ and high humidity (90\% RH) atmosphere, which was an environment inside a high-low temperature alternating damp heat cabinet (SDJ-402F, Chengdu Tianyu Co.).

\section{Stress-loading storage experiment}

5 screw cap assembly parts were stored at stress-loading experiment. At first the screw cap assembly part was assembled as initial state. Then a small pre-stress was applied on the screw cap part by rotating a screw tightly for $180^{\circ}$ from initial position, and then the intrinsic stress in the PC screw cap at the initial state were measured. The 5 screw cap assembly parts were then kept at this state and stored in normal laboratory environment (room temperature and middle humidity, 55-65\% RH). And the intrinsic stress and its distribution in each screw cap assembly part were measured after certain period of storage.

\section{Intrinsic stress determination method and instrumentation}

A photo-elastic analyzer was employed in determination of intrinsic stress and its distribution using Strain Matic M4/150.10 high resolution stress measuring system (Ilis company, Germany). The environmental condition for determination was controlled at $20 \pm 2^{\circ} \mathrm{C}$ and 50-60\% RH. The samples were stayed in the temperature-humidity environment in photo-elastic analyzer laboratory for 24 hours and approached to stable status before determination of the intrinsic stress and its distribution for polycarbonate samples. Multi-directional method with 6 measuring angles ( $15^{\circ}$ step width) in instrumental software was used to determine the intrinsic stress and its distribution. 


\section{Results and discussion}

Effect of environmental atmosphere storage on intrinsic stress and its distribution in PC screw cap assembly parts

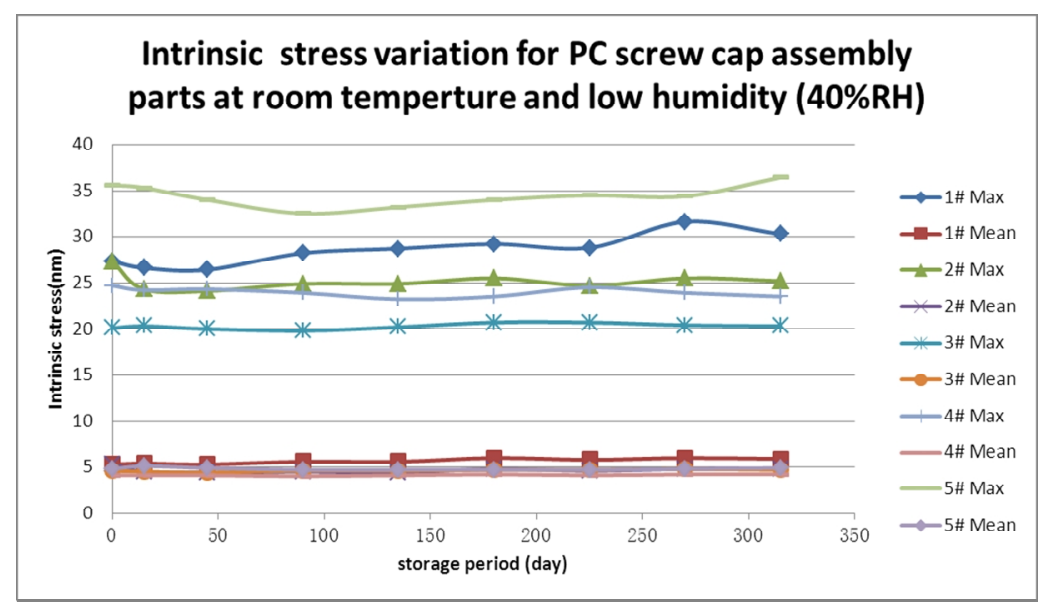

Figure 1 Room temperature and low humidity storage experiment

The result of room temperature and low humidity $(40 \% \mathrm{RH})$ storage experiment for 315 days was given in figure 1. After 315 days of storage at room temperature and low humidity environment, the maximal and mean intrinsic stress in screw cap assembly parts basically kept constant. The distribution of intrinsic stress also basically kept unchanged.

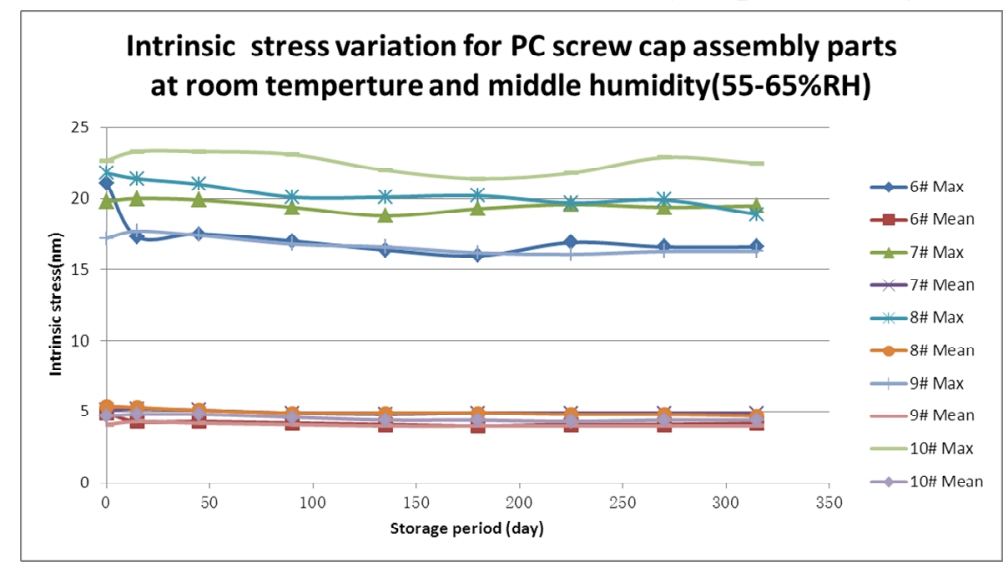

Figure 2 Room temperature and middle humidity storage experiment

The result of room temperature and middle humidity $(55-65 \% \mathrm{RH})$ storage experiment for 315 days was given in figure 2. After 315 days of storage at room temperature and middle humidity environment, the maximal and mean intrinsic stress in screw cap assembly parts only decreased by a little. The distribution of intrinsic stress basically kept unchanged.

The result of high temperature and high humidity $\left(47^{\circ} \mathrm{C}+90 \% \mathrm{RH}\right)$ storage experiment for 315 days was given in figure 3. After 315 days of storage at high temperature and high humidity environment, the maximal and mean intrinsic stress in screw cap assembly parts decreased obviously. The distribution of intrinsic stress basically kept unchanged.

The above storage experiments revealed that room temperature and dry environment basically kept the intrinsic stress in PC screw cap constant, while high temperature and high humidity environment lowered the intrinsic stress in PC screw cap obviously. High 
temperature and high humidity had effect similar to heat treatment, which promoted interior molecules to move and rearrange to lower the energy of system. The distribution of the intrinsic stress in PC screw cap basically kept unchanged during above environmental atmosphere storage.

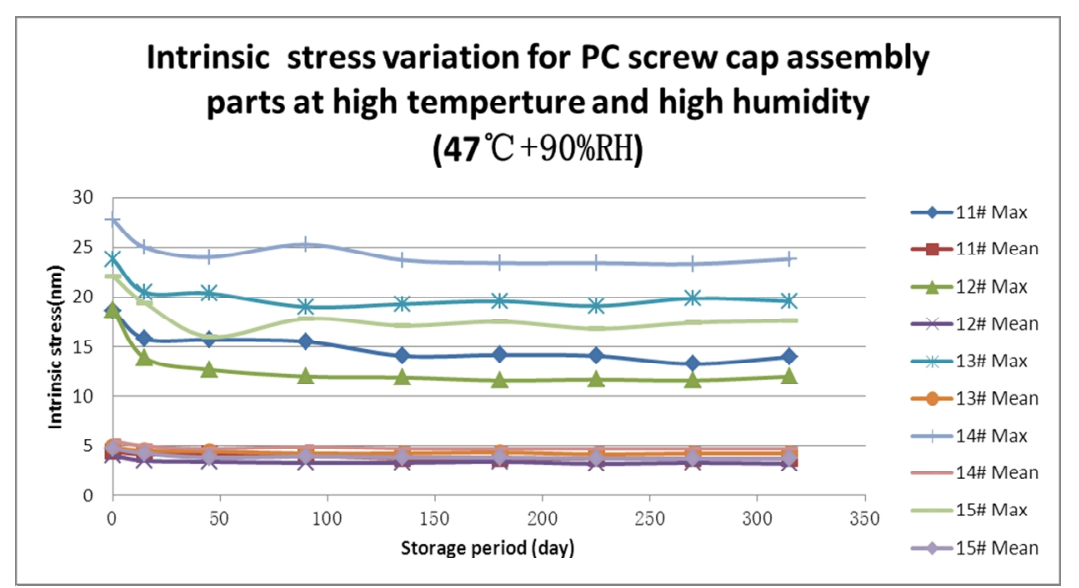

Figure 3 High temperature and high humidity $\left(47^{\circ} \mathrm{C}+90 \% \mathrm{RH}\right)$ storage experiment Effect of stress-loading storage on intrinsic stress and its distribution in PC screw cap assembly parts

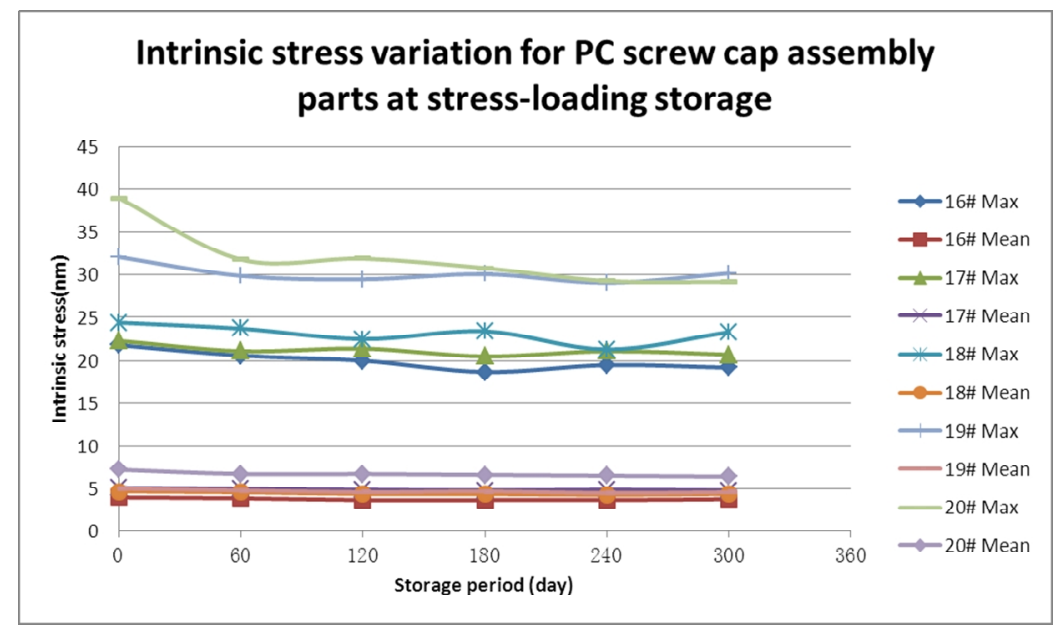

Figure 4 Stress-loading storage experiment

The results of stress-loading storage experiment for 5 screw cap assembly parts were given in figure 4. After 300 days of storage at stress loading (the screw was rotated tightly for $180^{\circ}$ ), the maximal and mean intrinsic stress in 5 screw cap assembly parts decreased obviously. This revealed that during long-term stress-loading storage, the intrinsic stress in screw cap parts might decrease gradually, which might be caused by stress relaxation. The distribution of the intrinsic stress in 5 PC screw caps basically kept unchanged during stress-loading storage experiment.

\section{Conclusion}

1 For PC screw cap assembly parts, dry environment basically kept the intrinsic stress constant, while high temperature and high humidity environment decreased the intrinsic stress obviously. 2 For PC screw cap assembly parts, after stress-loading storage, the intrinsic stress had a trend to 
decrease gradually, which might be caused by stress relaxation.

3 The distribution of intrinsic stress in polycarbonate screw cap part basically kept unchanged during environmental atmosphere storage and stress-loading storage.

\section{Acknowledgements}

The authors would like to gratefully acknowledge the Institute of Machinery Manufacturing Technology, China Academy of Engineering Physics for financial support.

\section{References}

1 Peter R. Lewis, Environmental stress cracking of polycarbonate catheter connectors, Engineering Failure Analysis, 2009, 16, 1816-1824.

2 Lutfi F. Al-Saidi et al., Environmental stress cracking resistance. Behavior of polycarbonate in different chemicals by determination of the time-dependence of stress at constant strains, Polymer Degradation and Stability, 2003,82,451-461.

3 R. Ramani, M. B. Ashalatha, A. Balraj, C. Ranganathaiah, Influence of gamma irradiation on the formation of methanol induced micro-cracks in polycarbonate, Journal of Materials Science, 2003, 38, 1431- 1438.

4 S. Pasta, Fatigue crack growth through particulate clusters in polycarbonate materials, Engineering Fracture Mechanics, 2011, 78, 397-411.

5 C. Y. Ding et al., The laminated cracking mechanism for polycarbonate thin-wall part, Polymer Materials Science and Engineering, 2008, 24(11), 115-118.

6 S. L. Jiang et al., Study on the environmental stress cracking resistance of polycarbonate, Plastic Industry, 2011, 39(5), 82-85, 112.

7 H. T. Wang et al., Effect of heat treatment on environmental stress cracking properties of polycarbonate, Application of engineering plastics, 2003, 31(12), 27-28.

8 J. Han et al., Effect of injection moulding processing on environmental stress cracking behavior of polycarbonate products, Chinese Plastics, 2010, 24(4), 76-79.

9 Pichet PINIT et al., Full-field determination of principal-stress directions using photoelasticity with plane-polarized RGB lights, Optical Review, 2005, 12(3), 228-232.

10 Yao-Ting Zhang et al., Branch-cutting algorithm for unwrapping photoelastic phase map with isotropic point, Optics and Lasers in Engineering, 2012, 50, 619-631.

11 M.P. Malezhik et al., Photoelastic determination of dynamic crack-tip stresses in an anisotropic plate, International Applied Mechanics, 2006,42(5), 574-581.

12 Tae Hyun Baek et al., Computer simulation of photoelastic fringe pattern for stress analysis, AsiaSim 2004, LNAI 3398, 2005,pp214-221.

13 Jeong-Hwan Nam et al., A study on the development of photoelastic experimental hybrid method for color omatics, Journal of Mechanical Science and Technology, 2011, 25(7), 1797-1809.

14 Che-Way Chang et al., Evaluation of residual stress in pre-stressed concrete material by digital image processing photoelastic coating and hole drilling method, Measurement, 2009, 42, 552-558. 